GANIT J. Bangladesh Math. Soc. (ISSN 1606-3694) 37 (2017) 15-27

\title{
HOMOGENEOUS AND INHOMOGENEOUS MAXWELL'S EQUATIONS IN TERMS OF HODGE STAR OPERATOR
}

\author{
Zakir Hossine ${ }^{1, *}$ and Md. Showkat $\mathrm{Ali}^{2}$ \\ ${ }^{1}$ Department of Mathematics, Bangladesh University of Engineering and Technology, \\ Dhaka-1000, Bangladesh \\ ${ }^{2}$ Department of Applied Mathematics, University of Dhaka, Dhaka 1000, Bangladesh \\ *Corresponding author: zakirhossainmath@gmail.com
}

Received 04.05.2016 Accepted 25.05.2017

\begin{abstract}
The main purpose of this work is to provide application of differential forms in physics. For this purpose, we describe differential forms, exterior algebra in details and then we express Maxwell's equations by using differential forms. In the theory of pseudo-Riemannian manifolds there will be an important operator, called Hodge Star Operator. Hodge Star Operator arises in the coordinate free formulation of Maxwell's equation in flat space-time. This operator is an important ingredient in the formulation of Stoke'stheorem.
\end{abstract}

Keywords: Homogeneous, Inhomogeneous, Hodge Star operator, differential forms.

\section{Introduction}

In mathematical field of differential geometry and tensor calculus, differential forms are an approach to multivariable calculus that is independent of co-ordinates. Differential forms[5] provide a unified approach in defining integrands over curves, surfaces, volumes and higher dimensional manifolds. The modern notion of differential forms as well as the idea of the differential form being the wedge product of the exterior derivative, forming an exterior algebra, was pioneered by ElieCartan[3].

\section{Differential Forms}

A differential $k$-form $\varphi$ is a sum of terms of the forms $A\left(x_{1}, x_{2}, \ldots, x_{n}\right) d x_{r_{1}} \wedge d x_{r_{2}} \wedge \ldots \wedge d x_{r_{k}}$. Addition of forms and multiplication of forms by functions, is defined in the usual way. Multiplication of forms is defined by "concatenation”; i.e.,

$$
\begin{aligned}
& \left(d x_{r_{1}} \wedge d x_{r_{2}} \wedge \ldots \wedge d x_{r_{k}}\right) \wedge\left(d x_{s_{1}} \wedge d x_{s_{2}} \wedge \ldots \wedge d x_{s_{k}}\right) \\
& =d x_{r_{1}} \wedge d x_{r_{2}} \wedge \ldots \wedge d x_{r_{k}} \wedge d x_{s_{1}} \wedge d x_{s_{2}} \wedge \ldots \wedge d x_{s_{k}}
\end{aligned}
$$

subject to the conditions $\quad d x_{i} \wedge d x_{j}=-d x_{j} \wedge d x_{i}$

$$
d x_{i} \wedge d x_{i}=0
$$

where $i$ and $j$ are between 1 and $n$. 


\section{Exterior Differentiation}

Definition: Exterior differentiationd is the operation taking a $C^{r} k$-form $\varphi$ (for $\geq 1$ ) to a $C^{r-1}(k+1)$-form $d \varphi$ defined by the following properties:

1) (Linearity) For constants $c_{1}$ and $c_{2}$ and forms $\varphi_{1}$ and $\varphi_{2}$

$$
d\left(c_{1} \varphi_{1}+c_{2} \varphi_{2}\right)=c_{1} d\left(\varphi_{1}\right)+c_{2} d\left(\varphi_{2}\right)
$$

2) For a 0 -form i.e., a function $\varphi=A\left(x_{1}, x_{2}, \ldots, x_{n}\right)$

$$
\begin{aligned}
& d \varphi=d A \\
& =A_{x_{1}}\left(x_{1}, x_{2}, \ldots, x_{n}\right) d x_{1}+\cdots+A_{x_{n}}\left(x_{1}, x_{2}, \ldots, x_{n}\right) d x_{n}
\end{aligned}
$$

3) For a $k$-form i.e., a function $\varphi=A\left(x_{1}, x_{2}, \ldots, x_{n}\right) d x_{r_{1}} \wedge d x_{r_{2}} \wedge \ldots \wedge d x_{r_{k}}$.

$$
d \varphi=(d A) \wedge\left(d x_{r_{1}} \wedge d x_{r_{2}} \wedge \ldots \wedge d x_{r_{k}}\right)
$$

\section{The Hodge Star Operator}

The binomial coefficient which represents the dimension of the space of $p$-forms $\Omega^{p}(M)$ is the number of ways of selecting $p$ (unordered) objects from a collection of $n$ objects. It is evident that

$$
\left(\begin{array}{l}
n \\
p
\end{array}\right)=\left(\begin{array}{c}
n \\
n-p
\end{array}\right)
$$

which means that there are as many $p$-forms as $(n-p)$-forms. In other words, there should be a way of converting $p$-forms to $(n-p)$-forms, for instance, 3-forms on 4-dimension can be converted to 1 -forms and vice versa. The operator that does this conversion is called the Hodge Star Operator [9].

Definition 4.1: The Hodge Star Operatoris the unique linear map on a semi-Riemannian manifold from $p$-forms to $(n-p)$-forms defined by

$$
*: \Omega^{p}(M) \rightarrow \Omega^{(n-p)}(M)
$$

such that for all $\xi, \eta \in \Omega^{p}(M)$,

$$
\wedge * \eta=\langle\xi, \eta\rangle d V
$$

This is an isomorphism between $p$-forms and $(n-p)$-forms, $* \eta$ is called the dual of $\eta$ and $d V=d x^{1} \wedge \ldots \wedge d x^{n}$ is the volume forms. Suppose that $d x^{1}, \ldots, d x^{n}$ are positively oriented orthonormal basis of 1 -forms on some chart $\left(U_{\alpha}, \phi_{\alpha}\right)$ on a manifold $M$. In particular $d V=d x^{1} \wedge$ $\ldots \wedge d x^{n}$.

Let $1 \leq i_{1}<\cdots<i_{p} \leq n$ be an ordered distinct increasing indices and let $j_{1}<\cdots<j_{n-p}$ be their complement in the set $\{1,2, \ldots, n\}$, then,

$$
d x^{i_{1}} \wedge \ldots \wedge d x^{i_{p}} \wedge d x^{j_{1}} \wedge \ldots \wedge d x^{j_{n-p}}=\operatorname{sng}(I) d x^{1} \wedge \ldots \wedge d x^{n}
$$


Where $\operatorname{sng}(I)$ is the sign of the permutation $i_{1}<, \ldots,<i_{p}$ in $\{1,2, \ldots, n\}$. In other words, the wedge products of $p$-forms and $(n-p)$-forms yields the volume form up to a sign. We claim that

$$
*\left(d x^{i_{1}} \wedge \ldots \wedge d x^{i_{p}}\right)=\operatorname{sgn}(I) \varepsilon_{i_{1}} \ldots \varepsilon_{i_{p}} d x^{j_{1}} \wedge \ldots \wedge d x^{j_{n-p}}
$$

Where $\operatorname{sgn}(I) \varepsilon_{i_{1}} \ldots \varepsilon_{i_{p}}= \pm 1$

Therefore, we have

$$
\begin{gathered}
* *\left(d x^{i_{1}} \wedge \ldots \wedge d x^{i_{p}}\right)=\operatorname{sgn}(I) \varepsilon_{i_{1}} \ldots \varepsilon_{i_{p}} \operatorname{sgn}(J) \varepsilon_{j_{1}} \ldots \varepsilon_{j_{n-p}} d x^{i_{1}} \wedge \ldots \wedge d x^{i_{p}} \\
=\operatorname{sgn}(I) \operatorname{sgn}(J) \prod_{k=1}^{n} \varepsilon_{k} d x^{i_{1}} \wedge \ldots \wedge d x^{i_{p}} \\
\Rightarrow *^{2}=(-1)^{p(n-p)+s}
\end{gathered}
$$

where $\prod_{k=1}^{n} \varepsilon_{k}=(-1)^{s}$ and $s$ is the signature of the metric.

The signature of the metric $s=0$ for Riemannian manifold and $s=1$ for Lorentzian manifold, thus,

$$
*^{2}=\left\{\begin{array}{l}
(-1)^{p(n-p)} \text { for Riemannian manifold } \\
(-1)^{p(n-p)+1} \text { for Lorentzian manifold }
\end{array}\right.
$$

We could rewrite (4.11) by introducing the totally anti-symmetric Levi-Civita permutation symbol defined by

$$
\epsilon_{i_{1}, \ldots, i_{p}}=\left\{\begin{array}{c}
+1 \quad \text { if }\left(i_{1}, \ldots, i_{p}\right) \text { is an even permutation of }(1,2, \ldots, n) \\
-1 \quad \text { if }\left(i_{1}, \ldots, i_{p}\right) \text { is an odd permutation of }(1,2, \ldots, n) \\
0 \quad \text { otherwise }
\end{array}\right.
$$

The Levi-Civita symbol [5] of all the indices up is equal to the permutation with all the indices down on Riemannian manifold,

$$
\epsilon_{i_{1}, \ldots, i_{p}}=\epsilon^{i_{1}, \ldots, i_{p}}
$$

Since the Riemannian metric [5] which is positive definite is used to raise or lower indices. However, this is not the case in Minkowski (Lorentzian manifold) 4-dimensional space-time, where index raising lowering is done with Minkowskimetric $\eta_{\mu \nu}$. Thus, in Minkowski 4dimensional space-time

$$
\epsilon_{i_{0} i_{1} i_{2} i_{3}}=-\epsilon^{i_{0} i_{1} i_{2} i_{3}},\left(\epsilon_{0123}=1\right),
$$

Since $\eta_{00}=-1$. The indices $i_{0}, i_{1}, i_{2}, i_{3}$ are any of the integers $0,1,2,3$. The important thing to note is that raising or lowering the index 0 introduces a negative sign. Using (4.13) we obtain

$$
*\left(d x^{i_{1}} \wedge \ldots \wedge d x^{i_{p}}\right)=\frac{1}{(n-p) !} \epsilon^{i_{1}, \ldots, i_{p}}{ }_{j_{1}, \ldots, j_{n-p}} d x^{j_{1}} \wedge \ldots \wedge d x^{j_{n-p}}
$$

Example 4.1 Suppose $d x^{1}, d x^{2}, d x^{3}$ are a basis of 1-forms on some chart $\left(U_{\alpha}, \phi_{\alpha}\right)$ on 3dimensional Riemannian manifold [9]. Then using (4.13) and (4.14) we obtain 


$$
\begin{gathered}
* d x^{1}=\frac{1}{2 !} \epsilon_{j_{1} j_{2}}^{1} d x^{j_{1}} \wedge d x^{j_{2}}=\frac{1}{2}\left(\epsilon_{23}^{1} d x^{2} \wedge d x^{3}+\epsilon_{32}^{1} d x^{3} \wedge d x^{2}\right)=\epsilon_{123} d x^{2} \wedge d x^{3}=d x^{2} \wedge d x^{3} \\
* d x^{2}=\frac{1}{2 !} \epsilon_{j_{1} j_{2}}^{2} d x^{j_{1}} \wedge d x^{j_{2}}=\frac{1}{2}\left(\epsilon_{13}^{2} d x^{1} \wedge d x^{3}+\epsilon_{31}^{2} d x^{3} \wedge d x^{1}\right)=-\epsilon_{123} d x^{1} \wedge d x^{3} \\
=-d x^{1} \wedge d x^{3} \\
* d x^{3}=\frac{1}{2 !} \epsilon_{j_{1} j_{2}}^{3} d x^{j_{1}} \wedge d x^{j_{2}}=\frac{1}{2}\left(\epsilon_{12}^{3} d x^{1} \wedge d x^{2}+\epsilon_{21}^{3} d x^{2} \wedge d x^{1}\right)=\epsilon_{123} d x^{1} \wedge d x^{2}=d x^{1} \wedge d x^{2}
\end{gathered}
$$

Conversely,

$$
\left.\begin{array}{c}
*\left(d x^{2} \wedge d x^{3}\right)=\frac{1}{1 !} \epsilon_{1}^{23} d x^{1}=d x^{1} \\
*\left(-d x^{1} \wedge d x^{3}\right)=\frac{1}{1 !} \epsilon_{2}^{13} d x^{2}=d x^{2} \\
*\left(d x^{1} \wedge d x^{2}\right)=\frac{1}{1 !} \epsilon_{3}^{12} d x^{3}=d x^{3}
\end{array}\right\}
$$

Suppose $d x^{0}, d x^{1}, d x^{2}, d x^{3}$ are a basis of 1-forms on some chart $\left(U_{\alpha}, \phi_{\alpha}\right)$ on 3-dimensional Minkowski space-time [6]. Then,

$$
\left.\begin{array}{l}
*\left(d x^{1} \wedge d x^{0}\right)=\frac{1}{2 !} \epsilon_{j_{1} j_{2}}^{10} d x^{j_{1}} \wedge d x^{j_{2}}=\epsilon_{0123} d x^{2} \wedge d x^{3}=d x^{2} \wedge d x^{3} \\
*\left(d x^{2} \wedge d x^{0}\right)=\frac{1}{2 !} \epsilon_{j_{1} j_{2}}^{20} d x^{j_{1}} \wedge d x^{j_{2}}=\epsilon_{0123} d x^{3} \wedge d x^{1}=d x^{3} \wedge d x^{1} \\
*\left(d x^{3} \wedge d x^{0}\right)=\frac{1}{2 !} \epsilon_{j_{1} j_{2}}^{30} d x^{j_{1}} \wedge d x^{j_{2}}=\epsilon_{0123} d x^{1} \wedge d x^{2}=d x^{1} \wedge d x^{2}
\end{array}\right\}
$$

Conversely,

$$
\left.\begin{array}{c}
*\left(d x^{2} \wedge d x^{3}\right)=\frac{1}{2 !} \epsilon_{j_{1} j_{2}}^{23} d x^{j_{1}} \wedge d x^{j_{2}}=-\epsilon_{0123} d x^{1} \wedge d x^{3}=-d x^{1} \wedge d x^{3} \\
*\left(d x^{3} \wedge d x^{1}\right)=\frac{1}{2 !} \epsilon_{j_{1} j_{2}}^{31} d x^{j_{1}} \wedge d x^{j_{2}}=\epsilon_{3120} d x^{2} \wedge d x^{0}=-d x^{2} \wedge d x^{0} \\
*\left(d x^{1} \wedge d x^{2}\right)=\frac{1}{2 !} \epsilon_{j_{1} j_{2}}^{12} d x^{j_{1}} \wedge d x^{j_{2}}=\epsilon_{1230} d x^{3} \wedge d x^{0}=-d x^{3} \wedge d x^{0}
\end{array}\right\}
$$

Notice something interesting in the above example, in 4-dimensional Minkowski space-time, the dual (Hodge Star Operator) of a 2-form is also a 2-form that is,

$$
*: \Omega^{2}(M) \rightarrow \Omega^{2}(M) \text {, with } *^{2}=-1
$$

The dual of a 3-form in 4-dimensional Minkowski space-time is given by

$$
\left.\begin{array}{l}
*\left(d x^{1} \wedge d x^{2} \wedge d x^{3}\right)=\epsilon_{0}^{123} d x^{0}=\epsilon_{1230} d x^{0}=-d x^{0} \\
*\left(d x^{0} \wedge d x^{1} \wedge d x^{3}\right)=\epsilon_{2}^{013} d x^{2}=-\epsilon_{0132} d x^{2}=d x^{2} \\
*\left(d x^{0} \wedge d x^{2} \wedge d x^{3}\right)=\epsilon_{1}^{023} d x^{1}=-\epsilon_{0231} d x^{1}=-d x^{1} \\
*\left(d x^{0} \wedge d x^{1} \wedge d x^{2}\right)=\epsilon_{3}^{012} d x^{3}=-\epsilon_{0123} d x^{3}=-d x^{3}
\end{array}\right\}
$$

Conversely,

$$
\left.\begin{array}{c}
* d x^{0}=\frac{1}{3 !} \epsilon_{j_{1} j_{2} j_{3}}^{0} d x^{j_{1}} \wedge d x^{j_{2}} \wedge d x^{j_{3}}=-\epsilon_{0123} d x^{1} \wedge d x^{2} \wedge d x^{3}=-d x^{1} \wedge d x^{2} \wedge d x^{3} \\
* d x^{1}=\frac{1}{3 !} \epsilon_{j_{1} j_{2} j_{3}}^{1} d x^{j_{1}} \wedge d x^{j_{2}} \wedge d x^{j_{3}}=\epsilon_{1023} d x^{0} \wedge d x^{2} \wedge d x^{3}=-d x^{0} \wedge d x^{2} \wedge d x^{3} \\
* d x^{2}=\frac{1}{3 !} \epsilon_{j_{1} j_{2} j_{3}}^{2} d x^{j_{1}} \wedge d x^{j_{2}} \wedge d x^{j_{3}}=\epsilon_{2013} d x^{0} \wedge d x^{1} \wedge d x^{3}=d x^{0} \wedge d x^{1} \wedge d x^{3} \\
* d x^{3}=\frac{1}{3 !} \epsilon_{j_{1} j_{2} j_{3}}^{3} d x^{j_{1}} \wedge d x^{j_{2}} \wedge d x^{j_{3}}=\epsilon_{3012} d x^{0} \wedge d x^{1} \wedge d x^{2}=-d x^{0} \wedge d x^{1} \wedge d x^{2}
\end{array}\right\}
$$


The exterior derivative [4] and Hodge Star Operator on $\mathbb{R}^{3}$ yield the known classical operators curl, divergence and gradient of vectors as we show now

Suppose $f$ is a 0 -form on $\mathbb{R}^{3}$. Then

$$
d f=\partial_{1} f d x^{1}+\partial_{2} f d x^{2}+\partial_{3} f d x^{3}
$$

If the coordinates are Cartesian, then the components are the components of the gradient of $f$. Thus,

$$
d f=\nabla f . d \boldsymbol{x}
$$

Let $A=A_{1} d x^{1}+A_{2} d x^{2}+A_{3} d x^{3}$ be a 1 -form on $\mathbb{R}^{3}$. Then

$$
\begin{gathered}
d A=\partial_{2} A_{1} d x^{2} \wedge d x^{1}+\partial_{3} A_{1} d x^{3} \wedge d x^{1}+\partial_{1} A_{2} d x^{1} \wedge d x^{2}+\partial_{3} A_{2} d x^{3} \wedge d x^{2} \\
+\partial_{1} A_{3} d x^{1} \wedge d x^{3}+\partial_{2} A_{3} d x^{2} \\
d A=\partial_{2} A_{1} d x^{2} \wedge d x^{1}+\partial_{3} A_{1} d x^{3} \wedge d x^{1}+\partial_{1} A_{2} d x^{1} \wedge d x^{2}+\partial_{3} A_{2} d x^{3} \wedge d x^{2} \\
+\partial_{1} A_{3} d x^{1} \wedge d x^{3}+\partial_{2} A_{3} d x^{2} \wedge d x^{3} \\
\left.\begin{array}{c}
\wedge d x^{3} \\
=\left(\partial_{1} A_{2}-\partial_{2} A_{1}\right) d x^{1} \wedge d x^{2}+\left(\partial_{1} A_{3}-\partial_{3} A_{1}\right) d x^{1} \wedge d x^{3}+\left(\partial_{2} A_{3}-\partial_{3} A_{2}\right) d x^{2} \wedge d x^{3} \\
* d A=\left(\partial_{2} A_{3}-\partial_{3} A_{2}\right) d x^{1}-\left(\partial_{1} A_{3}-\partial_{3} A_{1}\right) d x^{2}+\left(\partial_{1} A_{2}-\partial_{2} A_{1}\right) d x^{3}
\end{array}\right\}
\end{gathered}
$$

If the components are Cartesian, then the components are that of the curl a vector $\mathbf{A}$. That is,

$$
* d A=(\nabla \times \boldsymbol{A}) \cdot \boldsymbol{d} \boldsymbol{x}
$$

Notice that,

$$
\left.\begin{array}{c}
* A=A_{1} d x^{2} \wedge d x^{3}+A_{2} d x^{3} \wedge d x^{1}+A_{3} d x^{1} \wedge d x^{2} \\
d * A=\left(\partial_{1} A_{1}+\partial_{2} A_{2}+\partial_{3} A_{3}\right) d x^{1} \wedge d x^{2} \wedge d x^{3} \\
* d * A=\left(\partial_{1} A_{1}+\partial_{2} A_{2}+\partial_{3} A_{3}\right)=\nabla \cdot \boldsymbol{A} \text { in Cartesian coordinates. }
\end{array}\right\}
$$

\subsection{Equations of Electromagnetics}

The equations that relate the electromagnetics quantities will now be presented. Their proper introduction, in a textbook manner, should start with a description of the basic experiments (Coulomb, Ampere, Faraday, etc.): leading step by step to the final result using differential forms all along. This article does not allow enough space to do this properly. Therefore, we shall state the equations without other justification than their internal consistency and their agreement with the familiar vector calculus expressions.

The equations of electromagnetics are displayed in Tables I, II. All quantities are represented by form of various degrees, and they are designated by the letters conventionally used in the vector representation. The vector corresponding to a one-form is obtained by means of the over bar operator (e.g., $E \rightarrow \overrightarrow{\boldsymbol{E}}=\bar{E}$ ), while a vector corresponding to a two-form results from the star operator composed with the over bar

(e.g., $J \rightarrow \vec{J}=\overline{* J}$ ). Note that vectors corresponding to one-forms and two-forms are sometimes called polar and axial, respectively. This indicates different behavior under reflection which are obvious for differential forms submitted to a pullback under this operation. 
The equations decompose into two sets displayed in Table I (Maxwell-Faraday) and Table II (Maxwell-Ampere). In the upper part of these tables, diagonal arrows represent the operator $d$ (with respect to space variables) and horizontal arrows the time derivative $\partial_{t}$, or, for fields at frequency $\omega$, the product $-i \omega\left(e^{-i \omega t}\right.$ convention). (The arrows for $d$ go down in Table I, up in Table II.) A bar across an arrow means a negative sign. The quantity in any circle is the sum of those contributed by the arrows leading to it.

Thus, $B=a A, 0=d E+\partial_{t} B, E=-d \phi-d A$ etc.

Since $d . d=0, d B=0$ follows from $B=d A$.

Conversely (Poincare lemma), if $d B=0$ within a ball or a domain homeomorphic to it, there exists an $A$ in that domain such that $d A=B$.

Table I

Maxwell- Faraday Equations

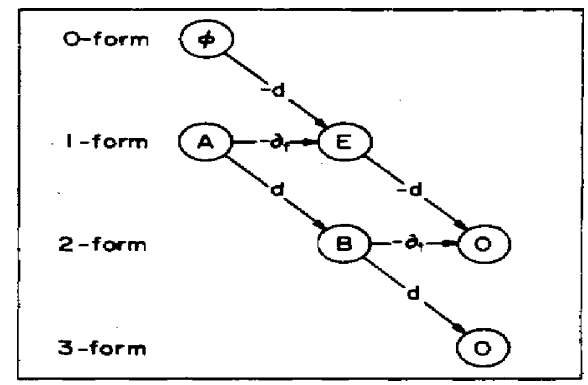

Space-Time Formulation

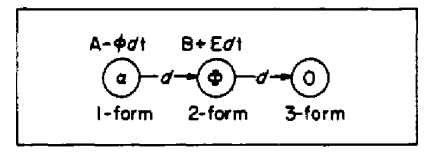

Table II

Maxwell-Ampere Equations

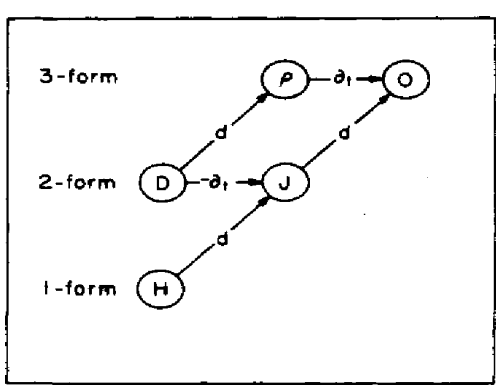

Space-Time Formulation

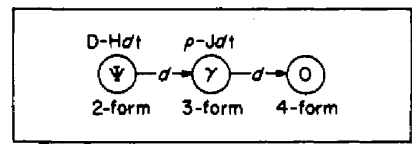

The equations in Tables I and II have the same expression in any system of coordinates. This cases to be true when the relations between the two tables are considered. With vector notations, this relation is expressed by

$$
\vec{D}=\epsilon_{0} \vec{E} \vec{B}=\mu_{0} \vec{H}
$$

where $\epsilon_{0}, \mu_{0}$ are the material constants, permittivity, and permeability.

In these equations, $\vec{E}$ and $\vec{H}$ correspond to one-forms $E$ and $H, \vec{D}$ and $\vec{B}$ to two-forms $D$ and $B$. The relation between the forms becomes

$$
D=\epsilon_{0} * E \text { and } B=\mu_{0}^{*} H
$$

where * is the star operator. We shall write

$$
D=\in E \quad B=\mu H
$$

Making $\in$ and $\mu$ into operators $\in_{0}{ }^{*}, \mu_{0}^{*}$, that include the star. 


\subsection{Stokes' Theorem in $\mathbb{R}^{3}$}

Let $S \subset \mathbb{R}^{3}$ be a smooth open surface bounded by a closed curve $C$. If $\vec{F}(x, y, z)$ be a continuous vector function which has continuous first partial derivatives in $S \subset \mathbb{R}^{3}$, then

$$
\oint_{c} \vec{F} \cdot d \vec{r}=\iint_{S}(\nabla \times \vec{F}) \cdot \widehat{n} d s
$$

where $\widehat{n}$ is the outward drawn unit normal vector to $S \subset \mathbb{R}^{3}$.

Using the differential form the above Stoke's theorem can be put in compact form as,

$$
\iint_{S} d \omega=\int_{\partial s} \omega
$$

where $S$, represents a surface in $\mathbb{R}^{3}$ and $\partial S$ represents its boundary ( a closed curve in $\mathbb{R}^{3}$ ).

\subsection{Generalized Stoke's Theorem}

Let $V^{p} \subset M^{n}$ be a compact oriented submanifold with boundary $\partial V$ in a manifold $M^{n}$. Let $\omega^{p-1}$ be a continuously differentiable $(p-1)$-form on $M^{n}$. Then

$$
\int_{V} d \omega^{p-1}=\int_{\partial V} \omega^{p-1}
$$

\section{Covariant Form of Maxwell's Equations}

Maxwell's equation [9] can be cast into covariant form. As Einstein expressed it "The general laws of nature are to be expressed by equations, which hold good for all systems of coordinates, that is, are covariant with respect to any substitution whatever.

Maxwell's theory of electromagnetism is, alongside with Einstein's theory of gravitation, one of the most beautiful of classical field theories. Having chosen units in which $\mu_{0}=\epsilon_{0}=c=1$, Maxwell's equations then take the form :

$$
\begin{aligned}
& \nabla . E=\rho \\
& \nabla \times B-\frac{\partial E}{\partial t}=J \\
& \nabla . B=0 \\
& \nabla \times E+\frac{\partial B}{\partial t}=0
\end{aligned}
$$

where, $E$ and $B$ are the electric and magnetic fields, $\rho$ and $J$ are the charge and current densities. Taking the divergence of equation (5.11) and substituting equation (5.12) into the resulting equation, we obtain the continuity equation

$$
\nabla . J+\frac{\partial \rho}{\partial t}=0
$$

Note that, we have used the fact that for any vector $\mathrm{H}$ and scalar $\mathrm{S}$

$$
\begin{gathered}
\nabla .(\nabla \times H)=0 \\
\nabla \times(\nabla S)=0
\end{gathered}
$$


Also, since equation (5.13) always holds, this means that $B$ must be a curl of a vector function, namely the vector potential $A$,

$$
B=\nabla \times A
$$

Substituting equation (5.16) into equation (5.14), we obtain

$$
\nabla \times\left(E+\frac{\partial A}{\partial t}\right)=0
$$

Which means that the quantity with vanishing curl in equation (5.17) can be written as the gradient of a scalar function, namely the scalar potential $\Phi$,

$$
E=-\nabla \Phi-\frac{\partial A}{\partial t}
$$

The minus sign attached to the gradient is for technical convenience. Thus, Maxwell's equation [6] can be written in covariant form by introducing the four vector potential $A^{\alpha}$ and the electric current four-vector potential $J^{\alpha}$ defined by

$$
\begin{aligned}
& A^{\alpha}=\left(\Phi, A^{1}, A^{2}, A^{3}\right)=\left(A^{0}, A^{1}, A^{2}, A^{3}\right) \\
& J^{\alpha}=\left(\rho, J^{1}, J^{2}, J^{3}\right)=\left(J^{0}, J^{1}, J^{2}, J^{3}\right)
\end{aligned}
$$

Equation (5.16) and (5.18) can then be written out explicitly in component form, for example

$$
\begin{aligned}
& B_{2}=\frac{\partial A^{1}}{\partial x^{3}}-\frac{\partial A^{3}}{\partial x^{1}}=\frac{\partial A^{1}}{\partial x_{3}}-\frac{\partial A^{3}}{\partial x_{1}}=\partial^{3} A^{1}-\partial^{1} A^{3} \\
& E_{1}=-\frac{\partial A^{0}}{\partial x^{1}}-\frac{\partial A^{1}}{\partial x^{0}}=\frac{\partial A^{1}}{\partial x_{0}}-\frac{\partial A^{0}}{\partial x_{1}}=\partial^{0} A^{1}-\partial^{1} A^{0}
\end{aligned}
$$

It is evident that the $E$ and $B$ fields are element of the second-rank, anti-symmetric, contravariant field-strength tensor $F^{\mu \nu}$ defined by

$$
F^{\alpha \beta}=\partial^{\alpha} A^{\beta}-\partial^{\beta} A^{\alpha}
$$

Explicitly, the field-strength is

$$
F^{\alpha \beta}=\left(\begin{array}{cccc}
0 & E_{1} & E_{2} & E_{3} \\
-E_{1} & 0 & B_{3} & -B_{2} \\
-E_{2} & -B_{3} & 0 & B_{1} \\
-E_{3} & B_{2} & -B_{1} & 0
\end{array}\right)
$$

where $\alpha$ corresponds to the rows and $\beta$ corresponds to the columns. The components of the fields in equations (5.16) and (5.18) can be easily identified as

$$
\begin{gathered}
E_{i}=F^{0 i} \\
B_{i}=\frac{1}{2} \epsilon^{i j k} F^{j k}, \quad i, j, k=1,2,3
\end{gathered}
$$

where the Levi-Civita symbol

$$
\epsilon^{i j k}=\left\{\begin{array}{ccc}
+1 & \text { if }(i, j, k)= & (1,2,3),(3,1,2),(2,3,1), \\
-1 & \text { if }(i, j, k)= & (1,3,2),(3,2,1),(2,1,3), \\
0 & \text { otherwise. }
\end{array}\right.
$$


It is very easy to show that the covariant field tensor defined by

$$
F_{\alpha \beta}=\partial_{\alpha} A_{\beta}-\partial_{\beta} A_{\alpha}
$$

has components

$$
F_{\alpha \beta}=\eta_{\alpha \gamma} \eta_{\beta \lambda} F^{\gamma \lambda}=\left(\begin{array}{cccc}
0 & -E_{1} & -E_{2} & -E_{3} \\
E_{1} & 0 & B_{3} & -B_{2} \\
E_{2} & -B_{3} & 0 & B_{1} \\
E_{3} & B_{2} & -B_{1} & 0
\end{array}\right)
$$

The Homogeneous Maxwell’s equations (5.13) and (5.14) correspond to the Jacobi identities

$$
\partial^{\gamma} F^{\alpha \beta}+\partial^{\alpha} F^{\beta \gamma}+\partial^{\beta} F^{\gamma \alpha}=0
$$

where $\alpha, \beta, \gamma$ are any of the integers $0,1,2,3$. For instance, if $\gamma=1, \alpha=2, \beta=3$ we have from equations (5.20) and (5.23)

$$
\partial^{1} F^{32}+\partial^{2} F^{13}+\partial^{3} F^{21}=-\left(\partial^{1} B_{1}+\partial^{2} B_{2}+\partial^{3} B_{3}\right)=-\left(\partial_{1} B_{1}+\partial_{2} B_{2}+\partial_{3} B_{3}\right)=0
$$

which indeed agrees with equation (5.13).

The Inhomogeneous Maxwell's equations [9] (5.11) and (5.12) can be written as

$$
\partial_{\beta} F^{\alpha \beta}=J^{\alpha}
$$

For instance,if $\alpha=0$, we have from (5.20) and (5.24)

$$
\partial_{0} F^{00}+\partial_{1} F^{01}+\partial_{2} F^{02}+\partial_{3} F^{03}=\partial_{1} E_{1}+\partial_{2} E_{2}+\partial_{3} E_{3}=\rho
$$

which indeed agrees with equation (5.12).

Notice that, the four Maxwell's equations have been reduced to a set of two equations (5.23) and (5.24). The continuity equation (5.15) was obtained from the inhomogeneous equations (5.11) and (5.12), similarly, the continuity equation in covariant form can be obtained from (5.24) by operating $\partial_{\mu}$ on both sides of equation (5.24). Thus,

$$
\partial_{\alpha} J^{\alpha}=\partial_{\alpha} \partial_{\beta} F^{\alpha \beta}=0
$$

Since $\partial_{\alpha} \partial_{\alpha}$ is symmetric in $\alpha$ and $\beta$ while $F^{\alpha \beta}$ is antisymmetric in $\alpha$ and $\beta$. The expression (5.26) is the conservation of electric charge whose underlying symmetry is gauge invariance.

\section{The Homogeneous Maxwell's Equation}

Having developed the mathematical language of differential forms [1], we hereby apply it to Maxwell's equations. First, consider the Homogeneous Maxwell's equations [9] (5.13) and (5.14), notice that in the language of differential forms, the divergence of a vector has been shown to be the exterior derivative of a 2-form on $\mathbb{R}^{3}$. The curl of a vector has also been shown to be the exterior derivative of 1 -form on $\mathbb{R}^{3}$. Thus, instead of treating the magnetic field as a vector $B=\left(B_{1}, B_{2}, B_{3}\right)$ we will treat it as a 2 -form

$$
B=B_{1} d x^{2} \wedge d x^{3}+B_{2} d x^{3} \wedge d x^{1}+B_{3} d x^{1} \wedge d x^{2}
$$


Similarly, instead of treating the electric field as a vector $E=\left(E_{1}, E_{2}, E_{3}\right)$, we will treat it as a 1form,

$$
E=E_{1} d x^{1}+E_{2} d x^{2}+E_{3} d x^{3}
$$

Next, we shall consider the electric and magnetic fields as the inhabitants of space-time and assume that the manifold $M$ to be a semi-Riemannian manifold [2] equipped with the Minkowski metric, in other words, as a 4-dimensional Lorenzian manifold or space-time. Furthermore, we shall assume that the space-time $M$ can be split into a 3-dimensional manifold $S$, space with a Riemannian metric and another space $\mathbb{R}$ for time. Then

$$
M=\mathbb{R} \times S
$$

Let $x^{i}(i=1,2,3)$ denote local coordinates on an open subset $U \subseteq S$, and let $x^{0}$ denote the coordinate on $\mathbb{R}$, then the local coordinates on $\mathbb{R} \times U \subseteq M$ will be those given by $x^{\alpha}=$ $\left(x^{0}, x^{1}, x^{2}, x^{3}\right)=(t, x)$ with the metric defined by

$$
\eta_{\alpha \beta}=\left(\begin{array}{cccc}
-1 & 0 & 0 & 0 \\
0 & 1 & 0 & 0 \\
0 & 0 & 1 & 0 \\
0 & 0 & 0 & 1
\end{array}\right)
$$

which is called flat metric tensor or flat Minkowski metric tensor [6], $\alpha, \beta=0,1,2,3$. We can now combine the electric and magnetic fields into a unified electromagnetic field $F$, which is a 2-form on $\mathbb{R} \times U \subseteq M$ defined by

$$
F=B+E \wedge d x^{0}(6.14)
$$

In component form, we have

$$
F=\frac{1}{2} F_{\alpha \beta} d x^{\alpha} \wedge d x^{\beta}
$$

Where $F_{\alpha \beta}$ is given by (5.22).

Explicitly, we have

$$
\begin{aligned}
& F=E_{1} d x^{1} \wedge d x^{0}+E_{2} d x^{2} \wedge d x^{0}+E_{3} d x^{3} \wedge d x^{0}+ \\
& B_{1} d x^{2} \wedge d x^{3}+B_{2} d x^{3} \wedge d x^{1}+B_{3} d x^{1} \wedge d x^{2}
\end{aligned}
$$

Taking the exterior derivative of (6.14) we obtain

$$
d F=d\left(B+E \wedge d x^{0}\right)=d B+d E \wedge d x^{0}
$$

In general, for any differential form $\eta$ on space-time, we have

$$
\eta=\eta_{I} d x^{I}
$$

where $I$ ranges over $i_{1}, i_{2}, \ldots, i_{p}$ and $\eta_{I}$ is a function of spacetime. Taking the exterior derivative [3] of (6.18), we obtain

$$
d \eta=\partial_{1} \eta_{I} d x^{1} \wedge d x^{I}+\partial_{2} \eta_{I} d x^{2} \wedge d x^{I}+\partial_{3} \eta_{I} d x^{3} \wedge d x^{I}+\partial_{0} \eta_{I} d x^{0} \wedge d x^{I}
$$




$$
\begin{array}{ll}
=\partial_{i} \eta_{I} d x^{i} \wedge d x^{I}+\partial_{0} \eta_{I} d x^{0} \wedge d x^{I}, & i=1,2,3 \\
& =d_{s} \eta+d x^{0} \wedge \partial_{0} \eta
\end{array}
$$

where $d_{s}$ is the exterior forms on a space-time, we shall split the exterior derivative into space-like part and time-like part. Using the identity above, we obtain the following from (6.17)

$$
\begin{aligned}
d F & =d_{s} B+d x^{0} \wedge \partial_{0} B+\left(d_{s} E+d x^{0} \wedge \partial_{0} E\right) \wedge d x^{0} \\
& =d_{s} B+d x^{0} \wedge \partial_{0} B+d_{s} E \wedge d x^{0}+d x^{0} \wedge \partial_{0} E \wedge d x^{0} \\
& =d_{s} B+\left(d_{s} E+\partial_{0} B\right) \wedge d x^{0}
\end{aligned}
$$

Now $d F=0$ is the same as

$$
\begin{aligned}
& d_{s} B=0 \\
& d_{s} E+\partial_{0} B=0
\end{aligned}
$$

The equation (6.19) and (6.20) are exactly the same as (5.13) and (5.14).

In order to be fully convinced that this is true [9], let's do the calculation explicitly in component form. Taking the exterior derivative of $F$ in (6.16), we obtain

$$
\begin{aligned}
& d F=\left(\partial_{1} B_{1}+\partial_{2} B_{2}+\partial_{3} B_{3}\right) d x^{1} \wedge d x^{2} \wedge d x^{3}+\left(\partial_{2} E_{3}-\partial_{3} E_{2}+\partial_{0} B_{1}\right) d x^{0} \wedge d x^{2} \wedge d x^{3} \\
& +\left(\partial_{3} E_{1}-\partial_{1} E_{3}+\partial_{0} B_{2}\right) d x^{0} \wedge d x^{3} \wedge d x^{1}+\left(\partial_{1} E_{2}-\partial_{2} E_{1}+\partial_{0} B_{3}\right) d x^{0} \wedge d x^{1} \wedge d x^{2}
\end{aligned}
$$

Note that $d F=0$ is the same as

$$
\begin{aligned}
& \partial_{1} B_{1}+\partial_{2} B_{2}+\partial_{3} B_{3}=0 \\
& \partial_{2} E_{3}-\partial_{3} E_{2}+\partial_{0} B_{1}=0 \\
& \partial_{3} E_{1}-\partial_{1} E_{3}+\partial_{0} B_{2}=0 \\
& \partial_{1} E_{2}-\partial_{2} E_{1}+\partial_{0} B_{3}=0
\end{aligned}
$$

The above four equations are exactly the same as (5.13) and (5.14). Hence, the Homogenous Maxwell's equations correspond to the closed form $d F=0$.

\section{The Inhomogeneous Maxwell's Equations}

In the old fashioned formulation of Maxwell's equations (see (5.12)-(5.14)), the Homogenous and the Inhomogeneous [9] versions are somehow related by reversing the role of $E$ and $B$. In the language of differential forms, this reversal relationship will lead to treating $E$ as a 2-form and $B$ as a1-form. Interestingly, the Hodge Star Operator does this work efficiently since one can easily convert a 1-form in 3-dimensional space to a 2-form and vice versa. Starting form (6.16) and using the results established in (4.16) and (4.17), we obtain

$$
* F=-B_{1} d x^{1} \wedge d x^{0}-B_{2} d x^{2} \wedge d x^{0}-B_{3} d x^{3} \wedge d x^{0}+
$$




$$
\begin{aligned}
& E_{1} d x^{2} \wedge d x^{3}+E_{2} d x^{3} \wedge d x^{1}+E_{3} d x^{1} \wedge d x^{2} \\
& \text { or* } F=\frac{1}{2}(* F)_{\alpha \beta} d x^{\alpha} \wedge d x^{\beta}
\end{aligned}
$$

where

$$
(* F)_{\alpha \beta}=\left(\begin{array}{cccc}
0 & B_{1} & B_{2} & B_{3} \\
-B_{1} & 0 & E_{3} & -E_{2} \\
-B_{2} & -E_{3} & 0 & E_{1} \\
-B_{3} & E_{2} & -E_{1} & 0
\end{array}\right)
$$

A close look at (6.16) and (7.11) shows that the effect of the dual operator on $F$ amounts to the exchange

$$
E_{i} \mapsto-B_{i} \text { and } B_{i} \mapsto E_{i}, i=1,2,3 \quad \text { in (5.22). }
$$

This is the main difference between the Homogeneous and the Inhomogeneous Maxwell's equations. Another difference is that the Inhomogeneous version contains $\rho$ and $J$. In the language of differential forms, we shall use the fact that the metric allows us to convert a vector field into a 1 -form. Combing the charge density and current density $J$ into a unified vector field on Minkowski space-time, we obtain

$$
J=J^{\alpha} \partial_{\alpha}=\rho \partial_{0}+J^{1} \partial_{1}+J^{2} \partial_{2}+J^{3} \partial_{3}
$$

withMinkowski metric (6.13), we obtain the 1 -form

$$
J=J_{\beta} d x^{\beta}=J^{1} d x^{1}+J^{2} d x^{2}+J^{3} d x^{3}-\rho d x^{0}
$$

where

$$
J_{\beta}=\eta_{\alpha \beta} J^{\alpha}
$$

Let $*_{s}$ denote the Hodge Star Operator on space, using (4.15) we can easily see that (7.11) is the same as

$$
* F=*_{s} E-*_{s} B \wedge d x^{0}
$$

which amounts to the exchange

$$
E \mapsto-*_{s} B \text { and } B \mapsto *_{s} E
$$

In (6.14), taking the exterior derivative of (7.17), we obtain

$$
d * F=d_{s} *_{s} E+\partial_{0} *_{s} E \wedge d x^{0}-d_{s} *_{s} B \wedge d x^{0}
$$

Applying Hodge Star Operator [5], we obtain

$$
* d * F=-*_{s} d_{s} *_{s} E \wedge d x^{0}-\partial_{0} E+*_{s} d_{s} *_{s} B
$$

If we set $* d * F=J$ and equate components, we obtain

$$
\begin{aligned}
& { }_{s} d_{s}{ }_{s} E=\rho \\
& -\partial_{0} E+{ }_{s} d_{s}{ }_{s} B=J^{i} d x^{i} i=1,2,3
\end{aligned}
$$

which is exactly the Inhomogeneous Maxwell's equation as can be shown explicitly by taking the exterior derivative of (7.11). 


$$
\begin{gathered}
d * F=\left(\partial_{1} E_{1}+\partial_{2} E_{2}+\partial_{3} E_{3}\right) d x^{1} \wedge d x^{2} \wedge d x^{3}+\left(\partial_{3} B_{2}-\partial_{2} B_{3}+\partial_{0} E_{1}\right) d x^{0} \wedge d x^{2} \wedge d x^{3} \\
+\left(\partial_{3} B_{1}-\partial_{1} B_{3}-\partial_{0} E_{2}\right) d x^{0} \wedge d x^{1} \wedge d x^{3}+\left(\partial_{2} B_{1}-\partial_{1} B_{2}+\partial_{0} E_{3}\right) d x^{0} \wedge d x^{1} \wedge d x^{2}
\end{gathered}
$$

Now $* d * F=J$ corresponds to

$$
\begin{aligned}
& \partial_{1} E_{1}+\partial_{2} E_{2}+\partial_{3} E_{3}=\rho \\
& \partial_{2} B_{3}-\partial_{3} B_{2}-\partial_{0} E_{1}=J^{1} \\
& \partial_{3} B_{1}-\partial_{1} B_{3}-\partial_{0} E_{2}=J^{2} \\
& \partial_{1} B_{2}-\partial_{2} B_{1}-\partial_{0} E_{3}=J^{3}
\end{aligned}
$$

Notice that, the above four equations are exactly the same as (5.11) and (5.12) and also $* d * F=J$ is similar to (5.24). Thus, the Maxwell's equations correspond to

$$
d F=0, * d * F=J .
$$

\section{Conclusion}

We know there are four classical Maxwell's equations. In our treatment we have been able to express them by two equations. We may expect that these will be useful in further research of Maxwell's equations.

\section{REFERENCES}

[1] Bachman David, A Geometric Approach to Differentials Forms, California Polytechnic State University, 2003.

[2] Boothby William M., An Introduction To Differentiable Manifolds and Riemannian Geometry, Academic Press, 2003.

[3] Chern, S.S., Chern, W.H., K.S. Law, Lectures on Differential Geometry, World Scientific Publishing Co. Pte. Ltd, 2000.

[4] Darling R.W.R., Differential Forms and Connections, Cambridge University Press, 1994.

[5] Frankel Theodore, The Geometry of Physics : An Introduction, Second edition, Cambridge University Press, 2006.

[6] Mikio Nakahara. Geometry, Topology and Physics, Institute of Physics Publishing, Second Edition, Bristol and Philadelphia, 2003.

[7] Reto Müller, Dr., Riemannian Geometry, Imperial College London, 2013.

[8] Reyer Sjamaar, Manifolds and Differential Forms. Department of Mathematics, Cornell University, Ithaca, New York, 2001.

[9] Solomon Akaraka Owerre, Maxwell's Equations in terms of Differential Forms, African Institute for Mathematical Sciences (AIMS), 2010. 